\title{
BMJ Open Clinical prediction rule for neurological sequelae due to acute encephalopathy: a medical community-based validation study in Harima, Japan
}

\author{
Kaori Sasaki, ${ }^{1}$ Hiroaki Nagase, ${ }^{2}$ Azusa Maruyama, ${ }^{3}$ Kyoko Fujita, ${ }^{4}$ \\ Masahiro Nishiyama, ${ }^{2}$ Tsukasa Tanaka, ${ }^{3}$ Sadayuki Nukina, ${ }^{5}$ Toru Takumi, ${ }^{6}$ \\ Kanae Takenaka, ${ }^{6}$ Yoshinobu Oyazato, ${ }^{1}$ Atsushi Nishiyama, ${ }^{1}$ Tomoko Kawata, ${ }^{7}$ \\ Keisuke Saeki, ${ }^{8}$ Yuichi Takami, ${ }^{9}$ Eriko Satake-Inoue, ${ }^{9}$ Kazumoto lijima, ${ }^{2}$ \\ Ichiro Morioka, ${ }^{2}$ Yoshiyuki Uetani ${ }^{4}$
}

To cite: Sasaki K, Nagase H, Maruyama A, et al. Clinical prediction rule for neurological sequelae due to acute encephalopathy: a medical communitybased validation study in Harima, Japan. BMJ Open 2017;7:e016675. doi:10.1136/ bmjopen-2017-016675

- Prepublication history and additional material for this paper are available online. To view these files, please visit the journal online (http://dx.doi org/10.1136/bmjopen-2017016675).

$\mathrm{HN}$ and IM contributed equally.

Received 2 March 2017 Revised 7 October 2017 Accepted 13 October 2017

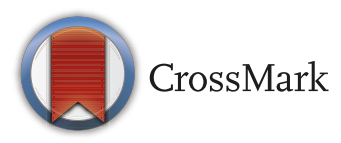

For numbered affiliations see end of article.

\section{Correspondence to}

Dr Hiroaki Nagase;

nagase@med.kobe-u.ac.jp and

Dr Ichiro Morioka;

ichim@med.kobe-u.ac.jp

\section{ABSTRACT}

Objectives This study aimed to verify the screening performance of our clinical prediction rule for neurological sequelae due to acute encephalopathy (NSAE-CPR), which previously identified the following three variables as predictive of poor outcomes: (1) refractory status epilepticus; (2) consciousness disturbance and/or hemiplegia at 6 hours from onset and (3) aspartate aminotransferase $>90 \mathrm{IU} / \mathrm{L}$ within 6 hours of onset. Design Medical community-based multicentre retrospective cohort study.

Setting Six regional hospitals in Harima and one tertiary centre in Kobe, Japan, from 2008 to 2012.

Participants We enrolled a total of 1612 patients aged $<16$ years who met the diagnostic criteria for an initial diagnosis of complex febrile seizure. Patients with a history of neurological disease and those included in the derivation cohort were excluded.

Primary outcome measures Univariate and multivariate analyses were performed to determine the association between each of the three predictor variables and poor $\mathrm{AE}$ outcome (Pediatric Cerebral Performance Category score $\geq 2$ ). Receiver operating characteristic curve (ROC) analysis was also performed to assess the screening performance of the NSAE-CPR.

Results The ROC analysis identified at least one of the three predictive variables as an optimal cut-off point, with an area under the curve of 0.915 (95\% $\mathrm{Cl} 0.825$ to 1.000). The sensitivity, specificity, positive and negative predictive values, positive and negative likelihood ratios and Matthews correlation coefficient were $0.867,0.954,0.149$, $0.999,18.704,0.140$ and 0.349 , respectively.

Conclusions Our findings indicate that the NSAE-CPR can be used for the screening and identification of patients with poor outcomes due to acute encephalopathy within 6 hours of onset.

\section{INTRODUCTION}

Children with acute encephalopathy (AE) and febrile seizure (FS) exhibit symptoms of seizure and/or altered consciousness, which
Strengths and limitations of this study

- This is the first published clinical prediction rule for neurological sequelae due to acute encephalopathy validated via a medical community-based multicentre retrospective cohort study, which may closely resemble the general population.

- Clinical symptoms such as altered consciousness may have been underestimated in some patients due to the retrospective design of the study.

- Several parameters associated with neurological outcomes such as duration of seizure and detailed treatments were not assessed.

are accompanied by fever without central nervous system (CNS) infection. ${ }^{1,2}$ FS is a primarily benign seizure condition in which patients typically experience good outcomes, and intensive therapeutic interventions are often unnecessary in patients with FS. In contrast, $\mathrm{AE}$ is diagnosed based on prolonged altered consciousness with/without seizure and is associated with a mortality rate of $6 \%$, according to a nationwide survey in Japanfar higher than that for FS. ${ }^{3}$ Furthermore, approximately $35 \%$ of patients with $\mathrm{AE}$ experience neurological sequelae such as an intellectual disability or vegetative state. ${ }^{4}$ Therefore, intensive therapeutic interventions are necessary for patients with AE. 5,6 Because patients with both AE and FS often present with seizure and altered consciousness early after onset, accurate differential diagnosis between $\mathrm{AE}$ and $\mathrm{FS}$ is critical for ensuring prompt initiation of intensive treatment for patients with AE. ${ }^{5}$ Some epidemiological studies have proposed that $\mathrm{AE}$ is defined as disturbed consciousness lasting longer than 24 hours. $^{78}$ However, as it is 
necessary to distinguish AE from FS within 24 hours of onset in clinical settings because the treatment methods are quite different, this definition is not clinically useful.

To address this issue, we previously conducted a singlecentre (Hyogo Prefectural Kobe Children's Hospital) retrospective study in which we identified three early risk factors for poor outcomes in this patient population: (1) refractory status epilepticus (RSE); (2) consciousness disturbance and/or hemiplegia at 6 hours from onset and (3) aspartate aminotransferase (AST) $>90$ IU/L within 6 hours from onset. A rule for the clinical prediction of neurological sequelae of $\mathrm{AE}$ within 6 hours of onset in patients at risk for poor outcomes (NSAE-CPR) was developed (derivation cohort) ${ }^{9}$ However, no studies have sought to validate the NSAE-CPR in other populations. The objective of the present study was to verify the screening performance of the NSAE-CPR in a medical community setting in Harima, Japan.

\section{PATIENTS AND METHODS}

\section{Study design and setting}

From January 2008 to December 2012, we conducted a multicentre retrospective cohort study involving six regional hospitals in Harima (Kakogawa Central City Hospital, Akashi City Hospital, Kita-Harima Medical Centre, St Mary's Hospital, Nishiwaki City Hospital and Japanese Red Cross Society Himeji Hospital) and one tertiary centre (Hyogo Prefectural Kobe Children's Hospital) in Kobe, Japan. The study was approved by the ethical committee of each participating facility, who waived the requirement for informed consent due to the retrospective nature of the study. The hospitals participating in this study accounted for $93.7 \%$ of individuals receiving inpatient care for $\mathrm{AE}$ or $\mathrm{FS}$ in this region in 2012 (validation cohort).

\section{Inclusion and exclusion criteria}

The present study used the same inclusion and exclusion criteria as the derivation study. ${ }^{9}$ FS was defined according to the guidelines provided by the National Institutes of Health consensus, with the exception of the age criterion. ${ }^{10}$ Patients under 16 years of age who met the diagnostic criteria for an initial diagnosis of complex FS were enrolled. Complex FS was defined when patients exhibited one or more of the following features: (1) partial onset or focal features during the seizure; (2) prolonged duration (>15 min) or (3) recurrent FS within 24 hours of the first episode. ${ }^{11}$ Patients who had a history of neurological conditions such as epilepsy, developmental delay, metabolic disorders or structural anomalies in the CNS were excluded. Patients included in the derivation study were also excluded.

\section{Definitions}

Onset time was defined as the time at which seizure or altered mental state was first described in the clinical chart. Blood tests were performed within 6 hours of onset. When two or more blood samples were available, the sample with the highest level of serum AST was chosen for subsequent analyses. RSE was defined as convulsive status epilepticus lasting longer than $60 \mathrm{~min}$ that failed to respond to first-line medications, such as diazepam. Disturbance of consciousness, defined as a Glasgow Coma Scale (GCS) score of 14 or lower, was diagnosed by the attending paediatricians and retrieved from each patient's medical record. Since altered mental state or hemiplegia cannot be evaluated during continuous treatment with anaesthetic drugs such as barbiturates or midazolam, data from patients exhibiting these conditions were considered to represent those of patients without altered mental state or hemiplegia for the analyses. Patients who met NSAE-CPR criteria were classified into a group at high-risk for poor prognosis, while those who did not meet NSAE-CPR criteria were classified into a low-risk group. Although AE is defined as prolonged impaired consciousness, it is sometimes difficult to evaluate the level of consciousness in children with $\mathrm{AE}$ in the clinical settings due to sedation. Therefore, in the present study, prolonged impaired consciousness due to $\mathrm{AE}$ was defined as disturbed consciousness lasting longer than 24 hours without sedatives. Patients who had prolonged impaired consciousness accompanied by fever without CNS infection or neurological sequelae there after were diagnosed with AE. Patients with conditions due to other aetiologies such as specific inborn error of metabolism were excluded.

\section{Prognosis}

Prognosis was evaluated 0-3 months from onset using the Pediatric Cerebral Performance Category Scale (PCPC). ${ }^{12}$ Children were classified into two groups based on PCPC score, as follows: good outcome $(\mathrm{PCPC}=1)$ and poor outcome $(\mathrm{PCPC}=2-6$ (2: mild sequelae 3: moderate sequelae 4 : severe sequelae 5 : vegetative state, 6 : brain death)).

\section{Study methods and statistical analyses}

We performed univariate analyses between each of the three predictor variables (RSE, consciousness disturbance or hemiplegia at 6 hours from onset and AST $>90$ $\mathrm{IU} / \mathrm{L}$ within 6 hours of onset $)^{9}$ and poor outcome in the validation cohort using Fisher's exact test. Variables significantly associated with poor outcome in the univariate analysis were further evaluated via multiple log-binomial analysis. To estimate the power of the NSAE-CPR, we calculated the screening performance of the NSAE-CPR in the validation and derivation cohorts (sensitivity, specificity, predictive values, likelihood ratios and Matthews correlation coefficient). Receiver operating characteristic curve (ROC) analysis was also performed to compare the area under the curve (AUC) between each cohort.

Several syndromes of $\mathrm{AE}$ characterised by variations in clinical course, neuroradiological findings and biochemical data have been established; such as acute encephalopathy with biphasic seizures and late reduced 
Table 1 Patient characteristics

\begin{tabular}{|c|c|c|}
\hline & $\begin{array}{l}\text { Derivation } \\
\text { cohort } n=86\end{array}$ & $\begin{array}{l}\text { Validation } \\
\text { cohort } \\
\mathrm{n}=1612\end{array}$ \\
\hline \multicolumn{3}{|l|}{ Age, months } \\
\hline Median (range) & $22(4-159)$ & $23(0-185)$ \\
\hline \multicolumn{3}{|l|}{ Gender, n (\%) } \\
\hline Male & $51(59.3)$ & $950(58.9)$ \\
\hline Poor outcome, n (\%) & $17(24.6)$ & $15(0.93)$ \\
\hline \multicolumn{3}{|l|}{ Predictor variables, n (\%) } \\
\hline Refractory status epilepticus & $27(31.4)$ & $52(3.2)$ \\
\hline $\begin{array}{l}\text { Consciousness disturbance } \\
\text { or hemiplegia at } 6 \text { hours from } \\
\text { onset }\end{array}$ & $13(15.1)$ & $35(2.2)$ \\
\hline $\begin{array}{l}\text { AST }>90 \text { IU/L within } 6 \text { hours } \\
\text { of onset }\end{array}$ & $5(5.8)$ & $10(0.62)$ \\
\hline $\begin{array}{l}\text { High-risk group (fulfilling any } \\
\text { of the predictor variables } \\
\text { above, NSAE-CPR) }\end{array}$ & $37(43.0)$ & $87(5.4)$ \\
\hline
\end{tabular}

AST, aspartate aminotransferase; NSAE-CPR, clinical prediction rule for neurological sequelae due to acute encephalopathy.

diffusion (AESD), ${ }^{13}$ acute necrotising encephalopathy, ${ }^{14}$ Reye syndrome ${ }^{15}$ and haemorrhagic shock and encephalopathy syndrome. ${ }^{16}$ Mizuguchi et al classified these syndromes into three major categories: metabolic error, systemic cytokine storm and excitotoxic neuronal injury (see online supplementary table 1). ${ }^{1}$ Our derivation study revealed that AST $>90 \mathrm{IU} / \mathrm{L}$ within 6 hours of onset predicts AE caused by metabolic error and/or cytokine storm, while normal AST levels are associated with excitotoxic neuronal injury. ${ }^{9}$ In the present study, we further evaluated the association between AST level and AE outcome to assess the screening performance for each category of AE.

All statistical analyses were performed using EZR (SaitamaMedical Center, JichiMedical University,
Saitama, Japan), which is a graphical user interface for R software (The R Foundation for Statistical Computing, Vienna, Austria). More precisely, it is a modified version of $\mathrm{R}$ commander designed to add statistical functions frequently used in biostatistics. A $\mathrm{P}$ value $<0.05$ was considered statistically significant.

\section{RESULTS \\ Patient characteristics}

The present study included a total of 1612 (median age: 23 months; $59 \%$ male) patients who met the criteria for an initial diagnosis of complex FS. Among these, 15 patients $(0.93 \%)$ experienced poor outcomes. RSE, consciousness disturbance or hemiplegia at 6 hours from onset and AST level $>90 \mathrm{IU} / \mathrm{L}$ within 6 hours of onset were observed in $52(3.2 \%), 35(2.2 \%)$ and 10 $(0.62 \%)$ patients, respectively. Eighty-seven patients $(5.4 \%)$ met the NSAE-CPR criteria and were classified into high-risk group (table 1).

\section{Univariate and multivariate analyses}

Univariate analysis revealed that all three predictive variables were significantly associated with poor outcome (RSE: $\mathrm{P}<0.0001$; consciousness disturbance or hemiplegia $\mathrm{P}=0.0001$; AST level $>90$ IU $/ \mathrm{L}: \mathrm{P}<0.0001)$. Multiple log-binomial analysis further revealed that RSE and level $>90$ IU/L within 6 hours of onset were independently associated with a poor outcome (RSE: relative risk=10.97 and $\mathrm{P}=0.0003$; AST level $>90 \mathrm{IU} / \mathrm{L}$ : relative risk $=16.12$ and $\mathrm{P}<0.0001$, table 2).

\section{ROC analysis}

The ROC analyses of the NSAE-CPR and poor outcome in the derivation and validation cohort identified at least one of the three predictive variables as an optimal cut-off point. The AUC values of the derivation and validation cohorts were 0.971 (95\% CI 0.913 to 1.000$)$ and 0.915 (95\% CI 0.825 to 1.000$)$, respectively.

Table 2 Univariate and multivariate analyses of the three predictor variables

\begin{tabular}{|c|c|c|c|c|}
\hline & $\begin{array}{l}\text { Good outcome } \\
n=1597\end{array}$ & $\begin{array}{l}\text { Poor } \\
\text { outcome } \\
n=15\end{array}$ & $\begin{array}{l}\text { Relative risk } \\
(95 \% \mathrm{Cl})\end{array}$ & P value \\
\hline \multicolumn{5}{|l|}{ Univariate analysis } \\
\hline Refractory status epilepticus, n (\%) & $44(2.76)$ & 8 (53.3) & 34.29 (12.92 to 90.99$)$ & $<0.0001$ \\
\hline $\begin{array}{l}\text { Consciousness disturbance or hemiplegia at } 6 \text { hours } \\
\text { from onset, } n(\%)\end{array}$ & $32(2.00)$ & $3(20.0)$ & 11.26 (3.33 to 38.15$)$ & 0.0001 \\
\hline \multicolumn{5}{|l|}{ Multivariate analysis } \\
\hline Refractory status epilepticus & & & 10.97 (2.97 to 40.47$)$ & 0.0003 \\
\hline $\begin{array}{l}\text { Consciousness disturbance or hemiplegia at } 6 \text { hours } \\
\text { from onset }\end{array}$ & & & $1.31(0.10$ to 17.76$)$ & 0.8047 \\
\hline AST >90 IU/L & & & $16.12(4.62$ to 56.26$)$ & $<0.0001$ \\
\hline
\end{tabular}

AST, aspartate aminotransferase. 
Table 3 Screening performance for the prediction of poor outcomes

\section{Derivation cohort $(\mathrm{n}=86)$}

\begin{tabular}{|c|c|c|c|c|c|}
\hline & Poor outcome & Good outcome & & Poor outcome & Good outcome \\
\hline \multirow[t]{2}{*}{ Low-risk group } & 1 & 48 & Low-risk group & 2 & 1523 \\
\hline & Values & $95 \% \mathrm{Cl}$ & & Values & $95 \% \mathrm{Cl}$ \\
\hline SENS & 0.941 & 0.713 to 0.999 & SENS & 0.867 & 0.595 to 0.983 \\
\hline SPEC & 0.696 & 0.573 to 0.801 & SPEC & 0.954 & 0.942 to 0.963 \\
\hline PPV & 0.432 & 0.271 to 0.605 & PPV & 0.149 & 0.082 to 0.242 \\
\hline NPV & 0.980 & 0.891 to 0.999 & NPV & 0.999 & 0.995 to 1.000 \\
\hline LR+ & 3.092 & 2.123 to 4.504 & LR+ & 18.704 & 13.881 to 25.201 \\
\hline LR- & 0.085 & 0.013 to 0.570 & LR- & 0.140 & 0.038 to 0.508 \\
\hline
\end{tabular}

LR-, negative likelihood ratio; LR+, positive likelihood ratio; MCC, Matthews correlation coefficient; NPV, negative predictive value; PPV, positive predictive value; PREV prevalence; SENS, sensitivity; SPEC, specificity.

\section{Screening performance}

The screening performance of the NSAE-CPR in the validation cohort is shown in table 3 . In the validation cohort, 13 (14.9\%) of 87 patients in the high-risk group experienced poor outcomes. The sensitivity, specificity, positive and negative predictive values, positive and negative likelihood ratios and Matthews correlation coefficient were as follows: $0.867,0.954,0.149,0.999$, $18.704,0.140$ and 0.349 respectively.

\section{Association between AST level and AE}

Thirty-eight patients were diagnosed with AE. Of these, detailed characteristics of the 15 patients who experienced poor outcomes are included in online supplementary table 2 . The pathomechapatients, excitotoxicity in six patients and undetermined in five. AST $>90 \mathrm{IU} / \mathrm{L}$ within 6 hours of onset was significantly associated with $\mathrm{AE}$ caused by cytokine storm (sensitivity $=1.000$, specificity $=0.996$, positive predictive value $=0.400$, negative predictive value $=1.000$, positive likelihood ratio $=268.000$, negative likelihood ratio $=0.140$ and Matthews correlation coefficient $=0.631, \mathrm{P}<0.001$; table 4). High-risk for poor prognosis without AST elevation was significantly associated with $\mathrm{AE}$ caused by excitotoxicity (sensitivity $=0.667$, specificity $=0.948$, positive predictive value $=0.046$, negative predictive value $=0.999$, nism of $\mathrm{AE}$ was identified as cytokine storm in four

Table 4 Association between AST level and acute encephalopathy caused by cytokine storm

\section{Derivation cohort $(n=86)$}

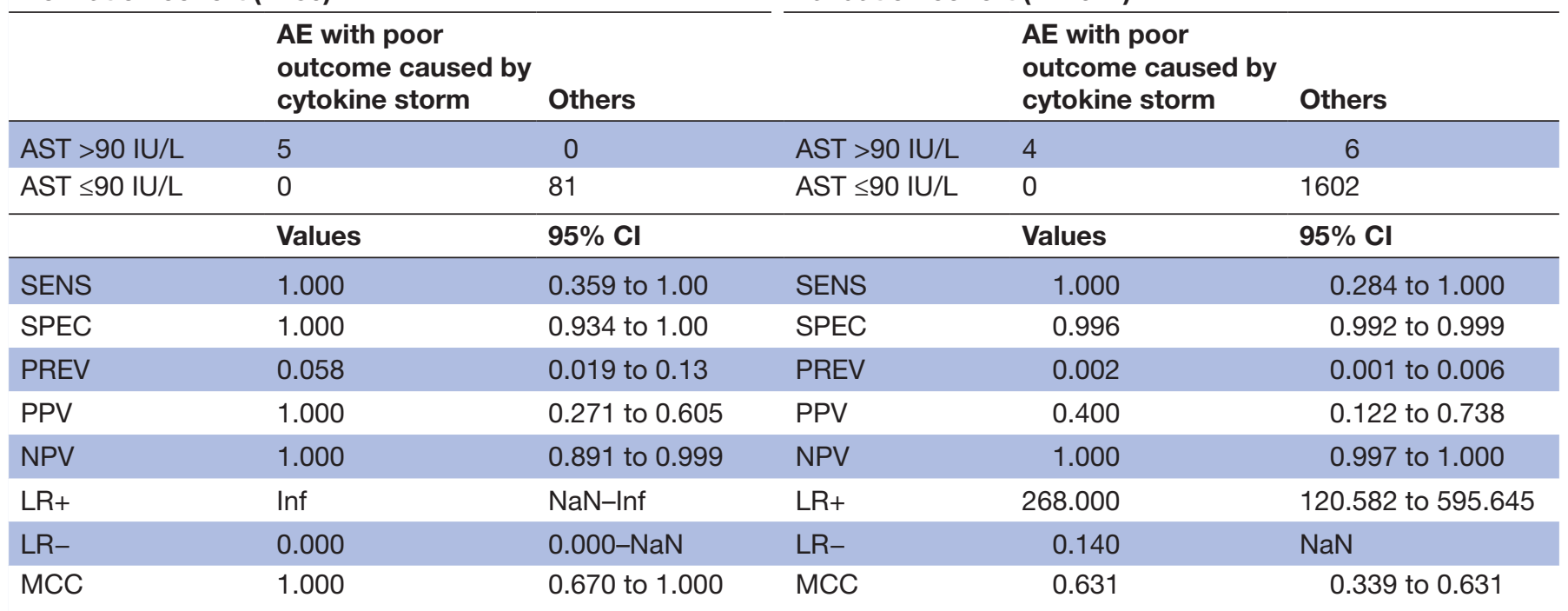

AE, acute encephalopathy; AST, aspartate aminotransferase; Inf, infinite; LR-, negative likelihood ratio; LR+, positive likelihood ratio; MCC, Matthews correlation coefficient; NPV, negative predictive value; NaN, not a number; PPV, positive predictive value; PREV prevalence; SENS, sensitivity; SPEC, specificity. 
Table 5 Association between AST $\leq 90 \mathrm{IU} / \mathrm{L}$ and acute encephalopathy due to excitotoxicity in the high-risk group

\begin{tabular}{|c|c|c|c|c|c|}
\hline \multicolumn{3}{|c|}{ Derivation cohort $(n=86)$} & \multicolumn{3}{|c|}{ Validation cohort $(n=1612)$} \\
\hline & $\begin{array}{l}\text { AE with poor } \\
\text { outcome } \\
\text { caused by } \\
\text { excitotoxicity }\end{array}$ & Others & & $\begin{array}{l}\text { AE with poor } \\
\text { outcome } \\
\text { caused by } \\
\text { excitotoxicity }\end{array}$ & Others \\
\hline $\begin{array}{l}\text { High-risk group with } \\
\text { AST } \leq 90 \text { IU/L }\end{array}$ & 11 & 1 & $\begin{array}{l}\text { High-risk group without } \\
\text { AST }>90 \mathrm{IU} / \mathrm{L}\end{array}$ & 4 & 83 \\
\hline & Values & $95 \% \mathrm{Cl}$ & & Values & $95 \% \mathrm{Cl}$ \\
\hline SENS & 0.917 & 0.615 to 0.998 & SENS & 0.667 & 0.223 to 0.957 \\
\hline SPEC & 0.986 & 0.927 to 1.000 & SPEC & 0.948 & 0.936 to 0.959 \\
\hline PREV & 0.140 & 0.074 to 0.231 & PREV & 0.004 & 0.001 to 0.008 \\
\hline PPV & 0.917 & 0.615 to 0.998 & PPV & 0.046 & 0.013 to 0.114 \\
\hline LR- & 0.084 & 0.0130 to 0.552 & LR- & 0.351 & 0.113 to 1.090 \\
\hline MCC & 0.903 & 0.682 to 0.973 & MCC & 0.166 & 0.067 to 0.230 \\
\hline
\end{tabular}

AE, acute encephalopathy; AST, aspartate aminotransferase; LR-, negative likelihood ratio; LR+, positive likelihood ratio; MCC, Matthews correlation coefficient; NPV, negative predictive value; PPV, positive predictive value; PREV prevalence; SENS, sensitivity; SPEC, specificity.

positive likelihood ratio=12.900, negative likelihood ratio $=0.351$ and Matthews correlation coefficient $=0.166, \mathrm{P}<0.001$; table 5 ).

\section{DISCUSSION}

Both AE and FS are clinically diagnosed based on seizure and/or consciousness disturbance with fever. Although it remains unknown whether $\mathrm{AE}$ and $\mathrm{FS}$ represent different syndromes or phenotypes of the same spectrum disorder, evidence suggests that the latter is more likely. The present study is the first medical community-based validation study of the NSAE-CPR. Our findings indicated that all three predictive variables identified in the derivation study were also significantly associated with poor outcome in the validation cohort. In addition, although we were unable to conduct a multivariate analysis in the derivation study because of the small sample size, the results obtained using the validation cohort confirmed that two of three variables were independently associated with poor outcome.

In the present study, we observed high sensitivity and specificity of the NSAE-CPR in the validation cohort, indicating that the NSAE-CPR is suitable for use as a screening tool and for identification of patients with poor outcomes. The NSAE-CPR was unable to accurately predict prognosis for 2 (13.3\%) of 15 patients in the validation cohort who experienced poor outcome, both of whom had been diagnosed with AESD. AESD is characterised by a biphasic clinical course: febrile status epilepticus early seizure) on the first day, followed by altered consciousness on the second day and clusters of brief seizures on the fourth or fifth day (late seizure). Since early seizures were brief, these two patients presented to the hospital following the appearance of late seizures, indicating that the NSAE-CPR may not be useful for patients who do not visit hospital immediately after onset.

AST elevation was observed in 6 of the 15 patients who experienced poor outcomes, 4 of whom were diagnosed with $\mathrm{AE}$ caused by cytokine storm. The results of our previous derivation study indicated that elevated AST level predicted this clinical situation with $100 \%$ sensitivity and specificity. ${ }^{9}$ Similarly, we also observed $100 \%$ sensitivity and $99.6 \%$ specificity in the present study, indicating that AST level $>90 \mathrm{IU} / \mathrm{L}$ within 6 hours of onset can be used to accurately identify and screen for AE caused by cytokine storm in patients with poor outcomes. Previous studies have revealed that corticosteroid therapy may be effective for AE caused by cytokine storm. ${ }^{5}$ However, further studies are required to evaluate the efficacy of corticosteroid therapy in high-risk patients with AST elevation.

Six out of nine patients in the poor outcome group with AST level $\leq 90 \mathrm{IU} / \mathrm{L}$ received final diagnoses of AESD, which is a common type of AE in Japan. Although sensitivity was not as high as that observed in the derivation study ( $66.7 \%$ vs $91.7 \%$ ), specificity was $94.8 \%$, suggesting that the NSAE-CPR can be used to identify AE caused by excitotoxicity in patients with poor outcomes.

\section{Strengths and limitations}

Patients in the derivation cohort of our previous study were recruited from a tertiary centre, while those in this validation cohort were recruited from multiple centres in the Harima region of Japan. Thus, the present cohort may closely resemble the general population. However, the present study also possesses some limitations of note. Clinical symptoms such as altered consciousness may have been underestimated in some patients due to the retrospective 
design of the study, which may have influenced diagnosis. The present study was designed as a validation study of our previous findings. ${ }^{9}$ The primary objective was to clarify whether the three clinical features associated with poor prognosis in our previous study ${ }^{9}$ are associated with poor prognosis in a medical community cohort likely to closely resemble the general population. A major limitation of our study was that several parameters associated with neurological outcomes such as symptoms of complex FS, duration of seizure and detailed treatments were not evaluated. Thus, further studies are indispensable.

Some patients with AE underwent specific forms of treatment. Pulsed steroid therapy, immunoglobulin therapy or edaravone treatment were performed in 28 , 12 and 6 patients, respectively. Four patients underwent targeted temperature management. Although these treatments may have influenced outcomes, treatments were chosen ad libitum for each patient in each facility, and patients with poor outcome received intensive treatments. To obtain more precise results for the validation of the NSAE-CPR, additional prospective studies are required. In addition, clinical trials should be conducted using the same protocol to assess the effects of specific symptoms and treatments. Nonetheless, the findings of the present study indicate that the NSAE-CPR is useful for the screening and identification of patients at risk for poor AE outcomes within 6 hours of onset, which may aid clinicians in making early decisions regarding intensive treatment. Our findings further indicate that AST level $>90 \mathrm{IU} / \mathrm{L}$ within 6 hours of onset can be used to identify patients at risk for poor outcomes associated with $\mathrm{AE}$ caused by cytokine storm.

\section{Author affiliations \\ ${ }^{1}$ Department of Pediatrics, Kakogawa Central City Hospital, Kakogawa, Japan \\ ${ }^{2}$ Department of Pediatrics, Kobe University Hospital, Kobe, Japan \\ ${ }^{3}$ Department of Neurology, Hyogo Prefectural Kobe Children's Hospital, Kobe, Japan \\ ${ }^{4}$ Emergency and General Mediciine, Hyogo Prefectural Kobe Children's Hospital, Kobe, Japan \\ ${ }^{5}$ Department of Pediatrics, Akashi City Hospital, Akashi, Japan \\ ${ }^{6}$ Department of Pediatrics, Kita-Harima Medical Center, Ono, Japan \\ ${ }^{7}$ Department of Pediatrics, St Mary's Hospital, Himeji, Japan \\ ${ }^{8}$ Department of Pediatrics, Nishiwaki City Hospital, Nishiwaki, Japan \\ ${ }^{9}$ Department of Pediatrics, Japanese Red Cross Society Himeji Hospital, Himeji, Japan}

Acknowledgements We thank Dr Takashi Omori (Professor of biostatistics, Department of Community Medicine and Social Healthcare Science, Kobe University Graduate School of Medicine) for his support of the statistical analyses.

Contributors KS and HN conceptualised and designed the study, carried out the initial analyses and drafted the initial manuscript. AM, KF, MN, TsT, SN, ToT, KT, YO, AN, TK, KS, YT and ES collected the patient data in this study. KI, IM and YU analysed the data and critically reviewed the manuscript. All authors approved the final manuscript as submitted and agree to be accountable for all aspects of the work.
Funding This work was supported by a grant from the Hyogo Prefectural Medical Association (MRF-H02-12). The funding organisation had no role in the design or conduct of this research.

\section{Competing interests None declared.}

Ethics approval The study was approved the ethical committee of each participating facility. Formal patient informed consent was waived because of the retrospective design.

Provenance and peer review Not commissioned; externally peer reviewed. Data sharing statement No additional data are available.

Open Access This is an Open Access article distributed in accordance with the Creative Commons Attribution Non Commercial (CC BY-NC 4.0) license, which permits others to distribute, remix, adapt, build upon this work non-commercially, and license their derivative works on different terms, provided the original work is properly cited and the use is non-commercial. See: http://creativecommons.org/ licenses/by-nc/4.0/

(C) Article author(s) (or their employer(s) unless otherwise stated in the text of the article) 2017. All rights reserved. No commercial use is permitted unless otherwise expressly granted.

\section{REFERENCES}

1. Mizuguchi M, Yamanouchi H, Ichiyama T, et al. Acute encephalopathy associated with influenza and other viral infections. Acta Neurol Scand Suppl 2007;186:45-56.

2. Guidelines for Epidemiologic Studies on Epilepsy. Commission on epidemiology and prognosis, international league against epilepsy. Epilepsia 1993;34:592-6.

3. Hoshino A, Saitoh M, Oka A, et al. Epidemiology of acute encephalopathy in Japan, with emphasis on the association of viruses and syndromes. Brain Dev 2012;34:337-43.

4. Nishiyama M, Nagase H, Tanaka T, et al. Short and long-term outcomes in children with suspected acute encephalopathy. Brain Dev 2016;38:731-7.

5. Okumura A, Mizuguchi M, Kidokoro $\mathrm{H}$, et al. Outcome of acute necrotizing encephalopathy in relation to treatment with corticosteroids and gammaglobulin. Brain Dev 2009;31:221-7.

6. Nishiyama M, Tanaka T, Fujita K, et al. Targeted temperature management of acute encephalopathy without AST elevation. Brain Dev 2015;37:328-33.

7. Amin R, Ford-Jones E, Richardson SE, et al. Acute childhood encephalitis and encephalopathy associated with influenza: a prospective 11-year review. Pediatr Infect Dis J 2008;27:390-5.

8. Centers for Disease Control and Prevention (CDC). Neurologic complications associated with novel influenza A (H1N1) virus infection in children - Dallas, Texas, May 2009. MMWR Morb Mortal Wkly Rep 2009;58:773-8.

9. Nagase H, Nakagawa T, Aoki K, et al. Therapeutic indicators of acute encephalopathy in patients with complex febrile seizures. Pediatr Int 2013;55:310-4.

10. Consensus statement. Febrile seizures: long-term management of children with fever-associated seizures. Pediatrics 1980;66:1009-12.

11. Nelson KB, Ellenberg JH. Prognosis in children with febrile seizures. Pediatrics 1978;61:720-7.

12. Fiser $\mathrm{DH}$. Assessing the outcome of pediatric intensive care. $J$ Pediatr 1992;121:68-74.

13. Takanashi J. Two newly proposed infectious encephalitis/ encephalopathy syndromes. Brain Dev 2009;31:521-8.

14. Mizuguchi M. Acute necrotizing encephalopathy of childhood: a novel form of acute encephalopathy prevalent in Japan and Taiwan. Brain Dev 1997;19:81-92.

15. Reye RD, Morgan G, Baral J. Encephalopathy and fatty degeneration of the viscera. A disease entity in childhood. Lancet 1963;2:749-52.

16. Levin M, Pincott JR, Hjelm M, et al. Hemorrhagic shock and encephalopathy: clinical, pathologic, and biochemical features. J Pediatr 1989;114:194-203. 